\title{
Virtual screening of natural compounds as combinatorial agents from indian medicinal plants against estrogen positive breast cancer
}

\author{
Shaleen Jain ${ }^{1}$, Dr. Asmita Das ${ }^{2}$ \\ ${ }^{1,2}$ Corresponding Author,Department of Biotechnology, Delhi Technological University, Main Bawana \\ Road, Delhi-110042 \\ Email: $\underline{\text { asmitadas1710@ dce.ac.in }}$ \\ asmita1710@gmail.com
}

\begin{abstract}
Facing worldwide challenges associated with multifactorial etiology of breast cancer, designing of combinatorial therapies using natural compounds is currently the emergent way of treating several cancers including breast cancer in a synergistic way, which may mitigate several problems associated with multiple receptor targeting. In this research, Estrogen receptor positive breast cancer was taken as prototype and several key receptors associated with this particular disease were targeted by virtual screening of natural compounds found in Indian originated medicinal plants using Computer aided Drug Designing (CADD) strategies. We found the combination of Carpusin, Paulownin Cornigerine, Nororientaline, Oryzalexin B, Romucosine $\mathrm{H}$ and Colchicine as effective against six potential receptors i.e. FGFR2, ESR1, PIK3CA, PIK3CB, PIK3CD and AR in Estrogen receptor positive breast cancer with their binding energies in the range of $\Delta \mathrm{G} \leq-8.0 \mathrm{Kcal} / \mathrm{mol}$ as well as significant number of common amino acid binding residues as compared with binding sites of receptors. Thus this research holds significant implications for the designing of combinatorial therapeutic agents against breast cancer which can be further tested in-vitro and in-vivo to prove their synergistic efficiency.
\end{abstract}

Key words: CADD, FGFR2, ESR1, Combinatorial therapies, PIK3, AR

\section{INTRODUCTION}

The occurrence of cancer is related to several factors to which human expose, the complexity of which is an arduous challenge in the field of epidemiology due to which many epidemiologists around the world have been understanding and determining cancer's multifactorial etiology. [1]

The complex interplay of several genetic and non-genetic factors have been clearly assessed in several researches that predispose to development of cancer. In this research, breast cancer was taken as a prototype due to its prevalence at a large scale worldwide and in major areas of India. Breast cancer has been described as multifactorial in occurrence in several epidemiological studies in which $90 \%$ cases occur due to the involvement of non-genetic factors, like environmental factors, major biological, chemical carcinogenic agents. [2]-[4]

To date, many researches have shown that a dysregulation of several molecular and signaling pathways occur due to the multifactorial effects pf breast cancer. Nevertheless, several potential therapies are needed for targeting multiple receptors and pathways to serve as an effective treatment against its proliferation and metastasis. [5]

Breast Cancer being an important concern of health and cause of deaths due to cancer among women worldwide has shown increased trends of prevalence and incidence, resulting in a high mortality rate globally. There has been a sharp increase in breast cancer cases in recent past. [6] [7]. According to 'Global Burden of Disease Cancer Collaboration', 2017, the data showed an increment by 33\% overall cancer cases from 2005 2015 worldwide. [8] [9]. The total cancer cases are likely to go up to 1,148,757 cases in 2020. [6]

The age standardized prevalence data of cancer cases shows 97 per one lakh individuals according to recent trends. According to a report by WHO, the present data of $6 \%$ total deaths due to cancer in India might be harmful in near future which is estimated to show an increment up to 9 million deaths by 2030. [9]-[11]

Improvements in cancer therapies have shown a remarkable success in recent past. Modern therapies have been developed which varies from DNA therapies based on DNA-excision repairing and modifying cellapoptotic pathways etc. to other potential treatments like hormone-based therapies like tamoxifen (Estrogen synthesis blocking agent) has been developed for breast cancer. Monoclonal antibody based treatment, Immune-based therapies (ICIs, CART therapies) etc. are other potential therapies. [12] [13]

Drug delivery systems like nanoparticles have also been developed in a past decade for targeted delivery in several cancers including breast cancer to avoid by-stander killing of tumor cells. [14] [15]. They include Doxorubicin, ThermoDox (Temperature based release of doxorubicin), SPIONs (Supermagnetic iron oxide nanoparticles), Nanotherm etc. [16] [17]

In the recent Covid-19 pandemic due to novel coronavirus (SARS COV-2), started out around December 2019, there has been a declination in cancer research worldwide and a high chances of increasing comorbidities in patients during this era. Cancer patients have been found vulnerable to Covid-19 infection according to some Chinese studies. Moreover consequences like delayed treatment, distraction in healthcare for cancer patients 
during pandemic conditions pose fatal consequences for cancer patients. [18]. Remarkable efforts have been made uptil now for overcoming the therapeutic and diagnostic challenges in this vulnerable population. According to recent safe guidelines of Canadian Association of Breast Imaging, delaying of breast cancer imaging and screening is needed to be avoid along with prevention of infection to the patients in Covid-19 pandemic. [18] [19]

Combinatorial therapies for cancer have been designed in many researches for overcoming challenges of cross-talk inhibition, signaling feedback etc. The rationale behind using multiple drugs is to suppress multiple receptors and pathways in a particular tumor to eradicate it in a synergistic way as compared with monotherapies. [20] [21]. Uptil now, different combinations of drugs including natural compound-based drugs, synthetic drugs, monoclonal antibodies etc. have been designed. [9] [22]. Examples include, USFDA approved Ipilimumab and nivolumab for unresectable melanoma. [23]. Combinatorial therapies have also been designed in Breast cancer. Eg. Combinations of Gemcitabine and Paclitaxel with Cyclophosphamide and Epirubicin in breast cancer. [24], combination of Fulvestrant and Anastrozole in hormone receptor positive breast cancer. [25].

Natural compounds has attained enormous benefits in therapeutics like stability, enhancement in efficacy, reduced toxicity, bioavailability, etc. In cancer treatment, many natural compound-based medicines have been designed as monotherapeutics and in combinations as well as in combination with synthetic compounds. [26]-[28]. Examples- Cyclophosphamide and Paclitaxel (Synthetic) in combination with curcumin (Natural), has been used in MDA-MB-231, MCF-12F and MCF-7 cancer cell lines. [29]-[33]. Combinations of natural compounds in breast cancer have been documented in several researches. [34] [31].

Nanoparticles in conjugation with natural compounds and their combinations have been tailored in different cancers including breast cancer, to act as an efficient targeted drug delivery tool to enhance the specificity and preventing bystander killing. [14]-[17].

\section{Breast Cancer as a prototype}

Breast Cancer has been found as the most frequently diagnosed cancer in India. The incidence rate has been estimated to $25.8 / 100000$ individuals. In this project, estrogen receptor positive breast cancer was taken as a prototype. Estrogen receptor positive breast cancer accounts for approximately $80 \%$ breast cancer cases. Uptil now, various therapies have been designed for ER positive BC. [35]. They include, endocrine therapies under which GnRHa (Gonadotropin releasing hormone agonists), SERMSs (Selective estrogen receptor modulators, eg. Tamoxifen), SERDs (Selective estrogen receptor down-regulators, eg. Fulvestrant) in combination with AIs (Aromatase inhibitors) are given as combinatorial drug therapies. [36] [37].

Although a vast majority of receptors are associated with the expression of $\mathrm{ER}(+) \mathrm{BC}$, but in this research, some potential receptors of $\mathrm{ER}(+) \mathrm{BC}$ in human were screened using a database which have been found to play an important role in its progression. Here, FGFR2 gene (encode protein from fibroblast growth receptor family), ESR1gene (encode estrogen receptor and a transcription factor), AR gene (encode androgen receptor), PIK3CA, PIK3CB and PIK3CD genes (encoding three catalytic isoforms i.e. p110 $\alpha$, p110 $\beta$ and p1 $10 \delta$ of phosphatidylinositol 4,5-bisphosphate 3-kinase respectively) were taken into consideration.

FGFRs play an important role in the progression of several cancers whose structures composed of different domains like 3 immunoglobulin-like domain, cytoplasmic tyrosine kinase domain, extra-cellular domain. [38]. FGFR2 has been reported as a crucial biomarker in breast cancer. In a research, its differently expressed genes (namely CXCL8, BMP7, CD44, MMP9) have been found between FGFR2 silenced breast cancer cell lines. FGFR2 also plays an important role in the progression of ER(+)BC. N550K and M5381 mutations of FGFR2 has been observed to offer resistance to CDK4/6, SERDs inhibitors in ER(+)BC patients. [38] [39]

ESR1 gene that encodes for estrogen receptor (ER) is an important biomarker in ER(+)BC which localizes to nucleus and forms homodimer or heterodimer with ESR2. [40]. In a research, patients with circulating ESR1 mutations have been found resistant to endocrine therapy which is given in ER BC treatment. In another research, these ESR1 mutations have been validated using ct-DNA (circulating tumor DNA) detection method in ER(+)BC. [41] [42]

Androgen receptor (encoded by AR gene) which have 3 functional domains and function as a transcription factor activated by steroid hormone act as a potential prognostic biomarker in breast cancers including $\mathrm{ER}(+) \mathrm{BC}$. [43]. Overexpression of $\mathrm{AR}$ as a key molecule has been seen in aromatase inhibitors (eg. exemestane) resistant $\mathrm{ER}(+) \mathrm{BC}$. Therefore, AR antagonism may serve as a promising strategy in $\mathrm{ER}(+) \mathrm{BC}$ which may in turn target several AR associated pathways like ERK1/2 signaling pathway, PI3K pathways etc. [44], [45]

The pathways of Phosphoinositide 3-kinase PIK (composed of a regulatory and catalytic subunits) shows an emerging prevalence and are responsible for frequent alterations in breast cancers including ER(+)BC. [46]. The three genes i.e. PIK3CA, PIK3CB and PIK3CD encode three catalytic isoforms i.e. $\mathrm{p} 110 \alpha, \mathrm{p} 110 \beta$ and $\mathrm{p} 110 \delta$ respectively and treatments using RNAi has been designed to target these isoforms. [47]-[49]. PIK3CA mutations have been reported crucial in fulvestrant resistant ER(+)BC using circulating tumor DNA (ct DNA), therefore PIK3CA has been found as an important regulator in progression and migration in $\mathrm{ER}(+) \mathrm{BC}$. [50]-[53]

In-silico approach in drug development 
Currently, In-silico approach has emerged as a promising strategy which ensures fruitful insights in the various fields like drug designing, target profiling, drug repurposing, polypharmacology, etc. [54]. Conventional pipeline remain centered on time-taking experiments and high delivery cost. Computer aided drug discovery (CADD) on other hand has exhibited as an effective technology for significant drug designing at a faster and cheaper rate in several diseases including cancers. [55]-[57]. Virtual screening, High throughput screening, Molecular docking etc. are emergent techniques for screening of drugs against particular protein receptors involved in various diseases as well as their structural and binding analysis. [58], [59]. Many useful tools have been developed for virtual screening and CADD. Autodock 4 [60], Autodock Vina [60], PyRx [61], PyMol [62], etc. are some of the open source online softwares available for studying the affinities between ligands and proteins and their binding poses, crystallographic analysis, molecular dynamics evaluation etc.

There are many databases available for ligand library screening like ZINC 15, PubChem, etc. IMPPAT (Indian Medicinal Plants, Phytochemistry and Therapeutics) is an online curated database (developed by The Institute of Mathematical Sciences, IMSc, India) which has many useful in-built webservers like admetSAR, FAF-Drugs4, FAF-QED, Open Babel, RDKit, etc. There is a huge library of 1742 medicinal plants of Indian origin, 9596 phytochemicals present in this database which have been used in traditional Indian medicines since time immemorial. [63]

\section{MATERIALS AND METHODS}

\section{Receptors selection}

510 genes associated with ER(+)BC were identified using DisGeNET, a curated database for genes associated with human diseases and variants. [64]. Then those 510 genes were filtered on the basis of Genedisease association score (Score gda $_{2} \geq 0.1$ ), probability of being loss of function intolerant ( $\mathrm{pLI} \geq 0.99$ ), total no. of PMIDs supporting association (N. PMIDs $\geq 7$ ) and identified top hits were further evaluated.

\section{Protein-Protein Interaction study}

Types of protein-protein interactions and their interaction scores between the selected receptors were calculated using STRING (11.0), a database for identification of proteins and their functional interactions. [65]. The strength of associations among them was evaluated from the number of nodes, PPI enrichment value, number of edges, average node degree, average local clustering coefficient and expected number of edges.

\section{Binding site analysis}

Binding sites of receptors were identified using Discovery Studio Visualizer (DSV), a software for analyzing sequences, modeling molecular structures and other data. [66], [67]. It locates all surrounding amino acid residues of a bounded ligand to the protein within a distance under defined threshold and forms a 2-D image which depicts all the amino acid residues and type of bonds through which they were attached to the ligand. In this research, high resolution crystal structures of proteins with their previously experimentally identified inhibitors/drugs were used and their drug sites were evaluated using DSV to which our concerned ligand was supposed to bind.

\section{Protein Dataset}

Minimum resolution X-ray crystallographic structures of FGFR2 (PDB Id: 3RI1- catalytic domain of fibroblast growth factor receptor 2 kinase in complex with ARQ069, Resolution- $2.10 \AA$, R-Value Free: 0.247), ESR1 (PDB Id: 5ACC- Estrogen receptor 1 in complex with AZD9496, Resolution- $2.10 \AA$, R-Value Free: 0.247), AR (PDB Id: 1E3G- Androgen receptor in complex with metribolone, Resolution- $2.10 \AA$, RValue Free: 0.247), PIK3CA (PDB Id: 3ZIM- Lipid kinase of Phosphatidylinositol 4,5-bisphosphate 3kinase catalytic subunit alpha isoform in complex with covalent inhibitor $\mathrm{C}_{30} \mathrm{H}_{35} \mathrm{~N}_{7} \mathrm{O}_{3} \mathrm{~S}$, Resolution$2.10 \AA$ Å, R-Value Free: 0.247), PIK3CB (PDB Id: 4BFR- Phosphatidylinositol 4,5-bisphosphate 3-kinase catalytic subunit beta isoform in complex with $\mathrm{C}_{19} \mathrm{H}_{22} \mathrm{~N}_{4} \mathrm{O}_{3}$, Resolution- $2.10 \AA$, R-Value Free: 0.247) and PIK3CD (PDB Id: 4XE0- Phosphatidylinositol 4,5-bisphosphate 3-kinase catalytic subunit delta isoform in complex with Idelalisib, Resolution- 2.10 $\AA$, R-Value Free: 0.247) were retrieved from PDB.

\section{Protein preparation}

Before docking of concerned ligands with selected proteins, proteins were prepared by deleting the hetatoms (co-crystallized water molecules, nonstandard residues, nonpolar hydrogens, small molecules and lone pairs) using DSV. Later gasteiger charges and polar hydrogens were added and merged using PyRx. Here, a file in pdbqt format is generated which is required for docking via Autodock.

\section{Ligand library preparation}

IMPPAT database was used for literature analysis of natural compounds using in-built webservers admetSAR, FAF-Drugs4, FAF-QED, RDKit. Initially all 9596 phytochemicals were primarily screened and filtered on the basis of zero Lipinski RO5 violations, zero Oral PhysChem Score (Traffic lights) which indicates good oral bioavailability, good GlaxoSmithKline's (GSK's) 4/400 score which indicates log P (octanol-water partition coefficient) $\leq 4$ and $\mathrm{M}_{\mathrm{w}}$ (Molecular weight) $\leq 400$ Dalton, Pfizer's 3/75 rule's good values which indicates small molecules with values of TPSA (Topological polar surface area) $>75$ and $\log P$ $<3 \AA^{2}$ indicating more drug likeliness and less toxicity, Egan rule's good values $(-1.0 \leq \operatorname{logP} \leq 5.8$ and TPSA $\leq 130 \AA^{2}$ ) which indicates good oral bioavailability, veber rule's good value (TPSA $<140$ and number of 
rotatable bonds $\leq 10$ ) which satisfies good oral bioavailability, good QEDw (Quantitative estimate of druglikeness) score $(\geq 0.8$ ) which evaluates the weighted geometric mean of log $\mathrm{P}$, number of hydrogen donors $(\leq 5)$ and acceptors $(\leq 10)$, molecular weight, No. Of rotatable bonds, TPSA, number of structural alerts and number of aromatic rings. QEDw $\geq 0.8$ signifies high druggability of compounds. Later, the primarily screened compounds were further secondarily screened on the basis of good PK properties like positive for BBB (Blood Brain Barrier) permeability, positive for HIA (Human intestinal absorption), good solubility. After screening, ligands were prepared finally for docking after energy minimization for 200 conjugate gradient steps with an UFF force field using PyRx.

\section{Molecular docking}

After secondary screening and preparation of ligands and receptors, they were subjected to structurebased drug designing through molecular docking using PyRx (with in-built Autodock tool). After selecting the binding sites manually, a grid box of dimension $60 \times 60 \times 60 \AA$ was generated with grid spacing $0.375 \AA$ which confines the active sites of protein to which the concerned ligand was supposed to bind within a specific shortened area. Then, after completion of docking within the specific sites, the binding energies i.e. $\Delta \mathrm{G}$ (in $\mathrm{Kcal} / \mathrm{mol}$ ) were noted and the compounds were selected on the basis of top hits/ autodock vina good scores. It is considered that lower the $\Delta \mathrm{G}$, more effective the binding is (in the range of $\Delta \mathrm{G} \leq-8.0 \mathrm{Kcal} / \mathrm{mol}$ ).

\section{Screening of drug likeliness and ADMET properties evaluation}

The drug likeliness was analyzed using admetSAR open-source tool. The docked ligands having good $\triangle \mathrm{G}$ were again filtered through extensive ADMET properties such as good PK and PD properties like positive BBB permeability, positive HIA, positive calcium carbonate permeability, Non-inhibition of P-glycoprotein protein substrate and inhibitors, Non-inhibition of CYP 450 (Cytochrome P450) substrate and inhibitors (including 2C9, 2D6, 3A4, 1A2, 2C9, 2D6, 2C19, 3A4), low CYP 450 inhibitory promiscuity (CYP IP), Nonames test toxicity. P-glycoproteins and CYP enzymes substrate play a fundamental role in metabolism of drugs, so their inhibition affects the drug likeliness property of drugs. After assessing binding scores, important ADMET properties, obtained drugs were subjected to binding amino acid residues evaluation using PyRx and PyMol and proposed as combinatorial therapeutic agents.

\section{Binding amino acid residues evaluation}

After molecular docking and extensive ADMET analysis, the binding amino acid residues of finally selected docked ligands and the type of bonds through which they are attached were observed by generating a 2-D image using DSV. Later PyMol was used for depicting the bounded ligands in the binding pocket of receptors.

\section{Results and discussion}

In this research, natural compounds from Indian medicinal plants were screened against potent receptors of ER(+)BC. Further, they were assessed on the basis of good ADMET properties. The 7 top natural compounds having excellent ADMET properties were found to have multi-targeting effects with efficient binding energies and similar binding residues against the binding pockets of selected receptors in $\mathrm{ER}(+) \mathrm{BC}$. (Work flow in figure: 1).

\section{Receptors selection}

The associated genes for ER(+)BC were screened using DisGenet in which we found total 510 associated genes, out of which 6 top genes (FGFR2, ESR1, PIK3CA, PIK3CB, PIK3CD, AR) were filtered on the basis of parameters like pLI, Scoregda, N.PMIDs as mentioned previously. These genes are basically responsible for translation of mentioned receptors/proteins. Moreover, it was found that these six genes were of 'kinase' and 'nuclear receptor' protein class and have a large number of other associated diseases. Table showing 6 potent receptors of ER(+)BC assessed using DisGenet database. All six genes were found to have DSIg (Disease specificity index) $\geq 0.29$, out of which FGFR2 showed highest DSIg $=0.38$. Their DPIg (Disease pleiotropy index) was found $\geq 0.8, \mathrm{pLi}$ 0.99 , Score $_{\text {gda }} \geq 0.1, \mathbf{E I}_{\text {gda }}$ (Evidence index) $\geq 0.9$, although out of 6, only FGFR2 and ESR1 were found to have 2 and 1 SNPs ( Single nucleotide polymorphisms) respectively. (Table 1). So, on the basis of above parameters, these 6 receptors were found potent to regulate the biological behavior of $\mathrm{ER}(+) \mathrm{BC}$, therefore proposed for further evaluation.

\section{Protein- protein interactions}

Types and strength of protein-protein associations between the selected 6 receptors were assessed using STRING (11.0) database (Figure 2) which defines PPI (protein-protein interaction) with confidence ranges for obtained data scores (low confidence scores <0.4; medium confidence: 0.4-0.7; high confidence: >0.7). All 6 were found to have firm interactions among themselves with significant interaction scores as mentioned in Table 2. Moreover, 6 nodes, 10 edges, average node degree $=$ 3.33 , average local clustering coefficient $=0.806,2$ expected number of edges and PPI enrichment p-value $=3.48 \mathrm{e}-05$ were found. These values are strong enough to elucidate the significant interactions among receptors. Therefore, targeting these receptors was considered very useful in targeting associated pathways simultaneously for an effective multi-targeted effect of drugs. These receptors were further evaluated for molecular docking simulations.

\section{Protein retrieval and binding sites evaluation}

Binding sites or inhibitory sites of proteins after retrieving their crystal structures from PDB in complex 
with different experimentally identified inhibitors were assessed through DSV. The surrounding amino acid residues (which are considered as binding sites) of bounded ligand were noted from DSV which generates a 2-D image of bounded inhibitor/ligand and its surrounding amino acid residues within a distance of threshold value. As shown in Figure: 3, FGFR2, ESR1, PIK3CA, PIK3CB, PIK3CD, AR were found to have 10, 15, 11, 10, 11, 10 residues respectively.

\section{Ligands screening}

In this research, natural compounds from medicinal plants of Indian origin were assessed through IMPPAT database of 9596 phytochemicals. Initially they were filtered using FAF-Drugs4 and FAFQED open-source cheminformatics platforms on the basis of LRo5 violations, Oral PhysChem score (traffic lights), GSK's 4/400, Pfizer's 3/75, Veber's rule, Egan's rule, QEDw as mentioned previously. At this stage, we found 99 natural compounds satisfying above mentioned properties. Then out of them, 42 Natural compounds were filtered on the basis of PK and PD properties (BBB permeability preferentialy) using admetSAR open source tool. (Table: 4-6)

5. Molecular docking evaluations

In this research, Structure-based Drug Designing (SBDD) through molecular docking was undertaken using PyRx (with in-built Autodock). After molecular docking of 42 ligands at the selected binding sites. Here, 25 natural compounds out of 42 were found to have promising binding energies in the range of $\Delta \mathrm{G} \leq-8.0 \mathrm{kcal} / \mathrm{mol}$ with RMSD lower bound and RMSD upper bound values $=0$. These 25 selected compounds were further evaluated through extensive ADMET analysis using admetSAR. (Table: 3-6)

\section{Prediction of ADMET properties}

After getting the screening done on the basis of $\Delta \mathrm{G}$ (in the range $\leq-8 \mathrm{kcal} / \mathrm{mol}$ ), 23 natural compounds were assessed for ADMET properties for identification of likelihood of compounds using admetSAR online server on the basis of CYP450 inhibitory promiscuity, ames toxicity, as mentioned before. Here, top 7 natural compounds were found to possess mentioned desired properties (preferentially CYP inhibitory promiscuity) as shown in table 6. On other hand, compounds like 4-Hydroxysesamin (CID: CID:16745513), Pinoresinol (CID:17750970), Irilone (CID:5281779) among 23 compounds were refuted besides having very good $\Delta \mathrm{G}$ score, due to not fulfilling the criteria of CYP inhibition. (Table: 3-6). Then these 7 natural compounds were finally selected and subjected for binding amino acid residues evaluation using DSV and PyMol, within the particular domain (kinase or nuclear receptor domain) of concerned receptor. (Figure: 4-9)

7. Molecular docking studies of receptors

Having good binding efficiencies (indicating good stability and longer half-life) with 6 receptors, promising ADMET properties and significant number of common interacting amino acid residues as compared with binding sites of receptors, 7 natural compounds compounds whose 2-D structures were taken from PubChem as shown in Figure 6, were finally proposed on the basis of receptor based drug screening or Structure-based Drug Designing (SBDD). Here, we found that among top 7 compounds, some of them showed highest binding energies (as shown in table 3 ), $\Delta \mathrm{G}$ (in the range $\leq-8 \mathrm{kcal} / \mathrm{mol}$ ), as well as significant number of common amino acid residues with binding AA residues of protein. (Figure: 4,5,7-9). Besides this, it was also observed that some ligands like Paulownin-CID:3084131 which showed $\Delta \mathrm{G}$ of $-9.7 \mathrm{Kcal} / \mathrm{mol}$ with PIK3CD receptor and $\Delta \mathrm{G}$ of 8.7 Kcal/mol with PIK3CA receptor; Cornigerine-CID:100188 which showed $\Delta \mathrm{G}$ of $-8.3 \mathrm{Kcal} / \mathrm{mol}$ with PIK3CD receptor were refuted as no common amino residues were found. The various types of molecular interactions (H-bonds, $\pi$-sigma, alkyl, $\pi$-alkyl, $\pi$-Sulfur, etc.) among the ligand and binding residues were also studied as shown in Tables (7-12). Finally proposed and their combinations are believed to be promising in inhibiting the potential receptors of $\mathrm{ER}(+) \mathrm{BC}$ in a multi-targeted way. Furthermore, their synergistic effects can be evaluated further in in-vitro studies on $\mathrm{ER}(+) \mathrm{BC}$ cell lines.

\section{Conclusion}

In this research, virtual screening of natural compounds found in Indian medicinal plants was done against the key receptors of Estrogen positive breast cancer. Ligand library was created using IMPPAT database followed by molecular docking simulations and ADMET predictions using PyRx and admetSAR tools respectively. We hereby conclude that the combination of Carpusin-CID:134369, Paulownin-CID:3084131, Cornigerine-CID:100188, Nororientaline-CID:440632, Oryzalexin B-CID:176496, Romucosine HCID:5320994 and Colchicine-CID:6167 are potential natural compounds against key receptors in clinical expression of Estrogen positive breast cancer. Among them, Carpusin, Paulownin, Oryzalexin B and colchicine showed excellent ADMET properties. These seven compounds which possess good PK, PD, Physiochemical properties as well as high drug likeliness shows efficient binding with these key receptors. Therefore, they can be further explored for their synergistic efficiency in molecular dynamics studies to prove their potential to act as combinatorial therapeutic agents against estrogen receptor positive breast cancer.

LIST OF FIGURES: 
1. Figure 1: Figure showing methodology of virtual screening used in research.

2. Figure 2: Protein-Protein interactions of selected receptors for ER(+)BC using STRING (11.0) database. Colored lines depict edges and types of interactions as shown in key alongside.

3. Figure 3: Binding sites of (A) FGFR2 (PDB Id: 3RI1 - catalytic domain of fibroblast growth factor receptor 2 kinase in complex with ARQ069), (B) ESR1 (PDB Id: 5ACC- Estrogen receptor 1 in complex with AZD9496), (C) PIK3CA (PDB Id: 3ZIM- Lipid kinase of Phosphatidylinositol 4,5bisphosphate 3-kinase catalytic subunit alpha isoform in complex with covalent inhibitor $\mathrm{C}_{30} \mathrm{H}_{35} \mathrm{~N}_{7} \mathrm{O}_{3} \mathrm{~S}$ ), (D) PIK3CB (PDB Id: 4BFR- Phosphatidylinositol 4,5-bisphosphate 3-kinase catalytic subunit beta isoform in complex with $\mathrm{C}_{19} \mathrm{H}_{22} \mathrm{~N}_{4} \mathrm{O}_{3}$ ), (E) PIK3CD (PDB Id: 4XE0- Phosphatidylinositol 4,5-bisphosphate 3-kinase catalytic subunit delta isoform in complex with ldelalisib), (F) AR (PDB Id: 1E3GAndrogen receptor in complex with metribolone).

4. Figure 4: Figure showing 2-D image of binding amino acid residues of ligands and 3-D molecular surface representation of binding pocket of FGFR2 receptor. Ligands are shown in stick format bounded in receptor's pocket (Green cyan). (A) Paulownin-CID:3084131, (B) CornigerineCID:100188, (C) Oryzalexin B-CID:176496.

5. Figure 5: Figure showing 2-D image of binding amino acid residues of ligands and 3-D molecular surface representation of binding pocket of ESR1 receptor. Ligands are shown in stick format bounded in receptor's pocket (Green). (A) Carpusin-CID:134369, (B) Nororientaline-CID:440632, (C) Oryzalexin B-CID:176496.

6. Figure 6: Chemical structures of natural compounds taken from PubChem, shown with their respective PubChem IDs and plants. (A) Carpusin-CID:134369 found in Petrocarpus marsupium and Rheum austral, (B) Paulownin-CID:3084131 found in Gmelina arborea, (C) CornigerineCID:100188 found in Gloriosa superba, (D) Nororientaline-CID:440632 found in Erythrina fusca, (E) Oryzalexin B-CID:176496 found in Oryza sativa, (F) Romucosine H-CID:5320994 found in Annona cherimola, (G) Colchicine-CID:6167 found in Colchicum autumnale, Colchicum luteum, Crocus sativus and Gloriosa superba.

7. Figure 7: Figure showing 2-D image of binding amino acid residues of ligands and 3-D molecular surface representation of binding pocket of PIK3CA receptor. Ligands are shown in stick format bounded in receptor's pocket (Yellow). (A) Cornigerine-CID:100188, (B) NororientalineCID:440632, (C) Romucosine H-CID:5320994

8. Figure 8: Figure showing 2-D image of binding amino acid residues of ligands and 3-D molecular surface representation of binding pocket of PIK3CD receptor. Ligands are shown in stick format bounded in receptor's pocket (Grey). (A) Romucosine H-CID:5320994, (B) Colchicine-CID:6167

9. Figure 9: Figure showing 2-D image of binding amino acid residues of ligands with their type of interactions and 3-D molecular surface representation of binding pocket of PIK3CB and AR receptors. Ligands are shown in stick format bounded in receptor's pocket (Golden yellow- PIK3CB, Red- AR). (A) Carpusin-CID:134369 with PIK3CB, (B) Carpusin-CID:134369 with AR.

\section{LIST OF TABLES:}

1. Table 1: Table: Table showing potential associated genes in Estrogen receptor positive breast cancer using DisGeNET database screened on the basis of parameters like pLI (Probability of gene disease association) $\geq 0.99$, Score $_{\text {gda }}$ (Score of gene disease association) $\geq 0.1$, N.PMIDs (No. of PMIDs) $\geq 7$. Abbreviations: DSIg (Disease specificity index), DPIg (Disease pleiotropy index), EI gda $_{\text {(Evidence }}$ index), EL gda (Evidence level), N_diseases g ( No. of associated diseases to gene), N. SNPsgda (No. of single nucleotide polymorphisms associated with gene).

2. Table 2: Protein-Protein interaction score obtained from STRING (11.0) database.

3. Table 3: Binding energy scores of 7 top ligands with selected receptors. Scores highlighted in red were selected as best ligands with top binding energies with significant number of common interacting amino acid residues with receptor' binding sites.

4. Table 4: Druggability properties of top 7 selected natural compounds. Computed via FAF-Drugs4 and FAF-QED.

5. Table 5: Physicochemical properties of top 7 selected ligands. Computed via FAF-Drugs4 and RDKit

6. Table 6: ADMET analysis of top 7 selected ligands. Computed via admetSAR.

7. Table 7: Types of molecular interactions of top 3 ligands with FGFR2. Binding amino acid residues highlighted in red indicate similarity with the binding sites of receptor.

8. Table 8: Types of molecular interactions of top 3 ligands with ESR1. Binding amino acid residues highlighted in red indicate similarity with the binding sites of receptor.

9. Table 9: Types of molecular interactions of top 3 ligands with PIK3CA. Binding amino acid residues highlighted in red indicate similarity with the binding sites of receptor.

10. Table 10: Types of molecular interactions of top 1 ligands with PIK3CB. Binding amino acid residues highlighted in red indicate similarity with the binding sites of receptor.

11. Table 11: Types of molecular interactions of top 2 ligands with PIK3CD. Binding amino acid residues highlighted in red indicate similarity with the binding sites of receptor. 
12. Table 12: Types of molecular interactions of top 3 ligands with AR. Binding amino acid residues highlighted in red indicate similarity with the binding sites of receptor.

\section{Abbreviations:}

ER(+)BC: Estrogen Receptor Positive Breast CANCER WHO: World Health Organization

DNA: Deoxyribonucleic acid

ICIs: Immune Checkpoint Inhibitors

CART: Chimeric Antigen Receptor T-Cell Therapy

SPIONs: Supermagnetic iron oxide nanoparticles

SARS COV-2: Severe Acute Respiratory Syndrome

Coronavirus 2

USFDA: United States Food and Drug Administration

GnRHa: Gonadotropin releasing hormone agonists

SERMSs: Selective estrogen receptor modulators

SERDs: Selective estrogen receptor down-regulators

FGFR2: Fibroblast Growth Factor Receptor 2

ESR1: Estrogen Receptor 1

PIK3CA: Phosphatidylinositol 4,5-bisphosphate 3-

kinase Catalytic subunit alpha

PIK3CB: Phosphatidylinositol 4,5-bisphosphate 3-

kinase Catalytic subunit beta

PIK3CD: Phosphatidylinositol 4,5-bisphosphate 3-

kinase Catalytic subunit delta

AR: Androgen Receptor

CXCL8: C-X-C Motif Chemokine Ligand 8

BMP7: Bone Morphogenetic Protein 7

MMP9: Matrix Metallopeptidase 9

CD44: A Multifunctional Cell Surface Adhesion

Receptor

ESR2: Estrogen Receptor 2

CDK 4/6: Cyclin-dependent kinase 4/6

ct-DNA: circulating tumor DNA

ERK1/2: Extracellular signal-regulated protein kinase 1/2

CADD: Computer aided drug discovery

IMSc: The Institute of Mathematical Sciences

IMPPAT: Indian Medicinal Plants, Phytochemistry and Therapeutics
PMID: Unique identifier number used in PubMed STRING: Search Tool for the Retrieval of Interacting Genes/Proteins

FAF-Drugs4: Free ADME-Tox Filtering Tool

QEDw: Weighted quantitative Estimate of Drug-likeness

DisGeNET: Database of gene-disease associations

Score $_{\text {gda: }}$ Gene-disease association score

pLI: Probability of being loss of function intolerant

N.PMIDs: No. of PMIDs)

DSIg: Disease specificity index),

DPIg: Disease pleiotropy index),

$\mathrm{EI}_{\text {gda: }}$ : Evidence index),

ELgda: Evidence level),

N_diseases g: No. of associated diseases to gene),

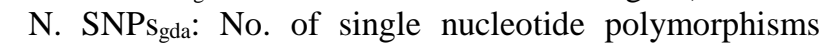
associated with gene).

DSV: Discovery Studio Visualizer

TPSA: Topological polar surface area

GSK: GlaxoSmithKline

$\log$ P: octanol-water partition coefficient

BBB: Blood Brain Barrier

HIA: Human intestinal absorption

ADMET: Absorption, Distribution, Metabolism, Excretion and Toxicity

CYP 450: Cytochrome P450

DSIg: Disease specificity index

DPIg: Disease pleiotropy index

PPI: Protein-Protein Interaction

PK: Pharmacokinetic properties

PD: Pharmacodynamic properties

RMSD: Root Mean Square Deviation

SBDD: Structure-based Drug Designing

LRo5: Lipinski Rule of five

LD50: Lethal Dose, 50\%

\section{Author's declaration:}

We authors of the above titled paper hereby declare that work included in the above paper is original and both authors share equal responsibilities.

\section{REFERENCES}

1. T. N. Toporcov and V. Wünsch Filho, "Epidemiological science and cancer control," Clinics, vol. 73, no. Suppl 1. Universidade de Sao Paulo, 2018, doi: 10.6061/clinics/2018/e627s.

2. Z. Momenimovahed and H. Salehiniya, "Epidemiological characteristics of and risk factors for breast cancer in the world," Breast Cancer: Targets and Therapy, vol. 11. Dove Medical Press Ltd., pp. 151164, 2019, doi: 10.2147/BCTT.S176070.

3. S. De Silva, K. H. Tennekoon, and E. H. Karunanayake, "Overview of the genetic basis toward early detection of breast cancer," Breast Cancer: Targets and Therapy, vol. 11. Dove Medical Press Ltd., pp. 71-80, 2019, doi: 10.2147/BCTT.S185870.

4. J. Clavel, "Progress in the epidemiological understanding of gene-environment interactions in major diseases: cancer," Comptes Rendus - Biologies, vol. 330, no. 4. Inserm, pp. 306-317, Apr. 2007, doi: 10.1016/j.crvi.2007.02.012.

5. M. L. Wan et al., "Colorectal cancer (CRC) as a multifactorial disease and its causal correlations with multiple signaling pathways," Bioscience Reports, vol. 40, no. 3. Portland Press Ltd, 2020, doi: 
10.1042/BSR20200265.

6. "Projections of number of cancer cases in India (2010-2020) by cancer groups - PubMed." https://pubmed.ncbi.nlm.nih.gov/21133622/ (accessed Aug. 16, 2020).

7. "WHO | Breast cancer: prevention and control," WHO, 2016, Accessed: Aug. 16, 2020. [Online]. Available: http://www.who.int/cancer/detection/breastcancer/en/.

8. N. Azamjah, Y. Soltan-Zadeh, and F. Zayeri, "Global trend of breast cancer mortality rate: A 25-year study," Asian Pacific J. Cancer Prev., vol. 20, no. 7, pp. 2015-2020, 2019, doi: 10.31557/APJCP.2019.20.7.2015.

9. S. Rajpal, A. Kumar, and W. Joe, "Economic burden of cancer in India: Evidence from crosssectional nationally representative household survey, 2014," PLoS One, vol. 13, no. 2, Feb. 2018, doi: 10.1371/journal.pone.0193320.

10. G. Mathew et al., "Community-based burden, warning signs, and risk factors of cancer using publicprivate partnership model in Kerala, India,” J. Fam. Med. Prim. Care, vol. 9, no. 2, p. 745, 2020, doi: 10.4103/jfmpc.jfmpc_1030_19.

11. P. Mathur et al., "Cancer Statistics, 2020: Report From National Cancer Registry Programme, India.," JCO Glob. Oncol., vol. 6, no. 6, pp. 1063-1075, Jul. 2020, doi: 10.1200/GO.20.00122.

12. J. Zhang, Y. Deng, and B. L. Khoo, "Fasting to enhance Cancer treatment in models: The next steps," Journal of Biomedical Science, vol. 27, no. 1. BioMed Central Ltd., May 05, 2020, doi: 10.1186/s12929020-00651-0.

13. K. L. Cheung, "Treatment strategies and survival outcomes in breast cancer," Cancers, vol. 12, no. 3. MDPI AG, Mar. 01, 2020, doi: 10.3390/cancers12030735.

14. S. Y. Wang, H. Z. Hu, X. C. Qing, Z. C. Zhang, and Z. W. Shao, "Recent advances of drug delivery nanocarriers in osteosarcoma treatment," Journal of Cancer, vol. 11, no. 1. Ivyspring International Publisher, pp. 69-82, 2020, doi: 10.7150/jca.36588.

15. W. Scarano, P. De Souza, and M. H. Stenzel, "Dual-drug delivery of curcumin and platinum drugs in polymeric micelles enhances the synergistic effects: A double act for the treatment of multidrugresistant cancer," Biomater. Sci., vol. 3, no. 1, pp. 163-174, Jan. 2015, doi: 10.1039/c4bm00272e.

16. Q. Mu et al., "Novel drug combination nanoparticles exhibit enhanced plasma exposure and doseresponsive effects on eliminating breast cancer lung metastasis," PLoS One, vol. 15, no. 3, 2020, doi: 10.1371/journal.pone.0228557.

17. J. Y. Choi, R. K. Thapa, C. S. Yong, and J. O. Kim, "Nanoparticle-based combination drug delivery systems for synergistic cancer treatment," Journal of Pharmaceutical Investigation, vol. 46, no. 4. Springer Netherlands, pp. 325-339, Jul. 01, 2016, doi: 10.1007/s40005-016-0252-1.

18. E. Moujaess, H. R. Kourie, and M. Ghosn, "Cancer patients and research during COVID-19 pandemic: A systematic review of current evidence," Critical Reviews in Oncology/Hematology, vol. 150. Elsevier Ireland Ltd, p. 102972, Jun. 01, 2020, doi: 10.1016/j.critrevonc.2020.102972.

19. H. Vatansev, C. Kadiyoran, M. Cumhur Cure, and E. Cure, "COVID-19 infection can cause chemotherapy resistance development in patients with breast cancer and tamoxifen may cause susceptibility to COVID-19 infection," Medical Hypotheses, vol. 143. Churchill Livingstone, p. 110091, Oct. 01, 2020, doi: 10.1016/j.mehy.2020.110091.

20. A. M. L. Seca and D. C. G. A. Pinto, "Plant secondary metabolites as anticancer agents: Successes in clinical trials and therapeutic application," International Journal of Molecular Sciences, vol. 19, no. 1. MDPI AG, Jan. 16, 2018, doi: 10.3390/ijms19010263.

21. A. Agbarya, N. Ruimi, R. Epelbaum, E. Ben-Arye, and J. Mahajna, "Natural products as potential cancer therapy enhancers: A preclinical update," SAGE Open Med., vol. 2, p. 205031211454692, Jan. 2014, doi: 10.1177/2050312114546924.

22. S. S. Bashraheel, A. Domling, and S. K. Goda, "Update on targeted cancer therapies, single or in combination, and their fine tuning for precision medicine," Biomedicine and Pharmacotherapy, vol. 125. Elsevier Masson SAS, May 01, 2020, doi: 10.1016/j.biopha.2020.110009.

23. A. F. Herrera et al., "Safety and activity of ibrutinib in combination with durvalumab in patients with relapsed or refractory follicular lymphoma or diffuse large B-cell lymphoma," Am. J. Hematol., vol. 95, no. 1, pp. 18-27, Jan. 2020, doi: 10.1002/ajh.25659.

24. F. A. Fisusi and E. O. Akala, "Drug Combinations in Breast Cancer Therapy," Pharm. Nanotechnol., vol. 7, no. 1, pp. 3-23, Jan. 2019, doi: 10.2174/2211738507666190122111224.

25. T. K. Burki, "Fulvestrant plus anastrozole for metastatic breast cancer," The Lancet. Oncology, vol. 20, no. 5. NLM (Medline), p. e247, May 01, 2019, doi: 10.1016/S1470-2045(19)30217-7.

26. K. H. Jung et al., "Targeted therapy of triple negative MDA-MB-468 breast cancer with curcumin delivered by epidermal growth factor-conjugated phospholipid nanoparticles," Oncol. Lett., vol. 15, no. 6, pp. 9093-9100, Jun. 2018, doi: 10.3892/ol.2018.8471.

27. B. Noel, S. K. Singh, J. W. Lillard, and R. Singh, "Role of natural compounds in preventing and treating breast cancer," Front. Biosci. - Sch., vol. 12, no. 1, pp. 137-160, Mar. 2020, doi: 10.2741/S544.

28. A. Lodi et al., "Combinatorial treatment with natural compounds in prostate cancer inhibits prostate tumor growth and leads to key modulations of cancer cell metabolism," npj Precis. Oncol., vol. 1, no. 1, 
Dec. 2017, doi: 10.1038/s41698-017-0024-z.

29. V. Shriram, T. Khare, R. Bhagwat, R. Shukla, and V. Kumar, "Inhibiting bacterial drug efflux pumps via phyto-therapeutics to combat threatening antimicrobial resistance," Front. Microbiol., vol. 9, no. DEC, Dec. 2018, doi: 10.3389/fmicb.2018.02990.

30. I. Górniak, R. Bartoszewski, and J. Króliczewski, "Comprehensive review of antimicrobial activities of plant flavonoids," Phytochemistry Reviews, vol. 18, no. 1. Springer Netherlands, pp. 241-272, Feb. 15, 2019, doi: 10.1007/s11101-018-9591-z.

31. L. Wang et al., "Wnt1-inducible signaling protein 1 regulates laryngeal squamous cell carcinoma glycolysis and chemoresistance via the YAP1/TEAD1/GLUT1 pathway,” J. Cell. Physiol., vol. 234, no. 9, pp. 15941-15950, Sep. 2019, doi: 10.1002/jcp.28253.

32. A. Moore and R. Pinkerton, "Vincristine: Can its therapeutic index be enhanced?," Pediatr. Blood Cancer, vol. 53, no. 7, pp. 1180-1187, Dec. 2009, doi: 10.1002/pbc.22161.

33. S. R. Lin et al., "Natural compounds as potential adjuvants to cancer therapy: Preclinical evidence," British Journal of Pharmacology, vol. 177, no. 6. John Wiley and Sons Inc., pp. 1409-1423, Mar. 01, 2020, doi: 10.1111/bph.14816.

34. S. Mitra and R. Dash, "Natural Products for the Management and Prevention of Breast Cancer," Evidence-based Complementary and Alternative Medicine, vol. 2018. Hindawi Limited, 2018, doi: $10.1155 / 2018 / 8324696$.

35. R. Dembinski et al., "Estrogen Receptor Positive and Progesterone Receptor Negative Breast Cancer: the Role of Hormone Therapy," Horm. Cancer, vol. 11, no. 3-4, pp. 148-154, Aug. 2020, doi: 10.1007/s12672-020-00387-1.

36. F. Lumachi, "Current medical treatment of estrogen receptor-positive breast cancer," World J. Biol. Chem., vol. 6, no. 3, p. 231, 2015, doi: 10.4331/wjbc.v6.i3.231.

37. D. Lüftner et al., "Update Breast Cancer 2020 Part 2 - Advanced Breast Cancer: New Treatments and Implementation of Therapies with Companion Diagnostics," Geburtshilfe Frauenheilkd., vol. 80, no. 4, pp. 391-398, Apr. 2020, doi: 10.1055/a-1111-8775.

38. "FGFR2 fibroblast growth factor receptor 2 [Homo sapiens (human)] - Gene - NCBI." https://www.ncbi.nlm.nih.gov/gene/2263 (accessed Aug. 14, 2020).

39. S. Navid, C. Fan, P. O. Flores-Villanueva, D. Generali, and Y. Li, "The fibroblast growth factor receptors in breast cancer: From oncogenesis to better treatments," International Journal of Molecular Sciences, vol. 21, no. 6. MDPI AG, Mar. 02, 2020, doi: 10.3390/ijms21062011.

40. "ESR1 estrogen receptor 1 [Homo sapiens (human)] - Gene - NCBI." https://www.ncbi.nlm.nih.gov/gene/2099 (accessed Aug. 14, 2020).

41. F. Clatot et al., "Risk of early progression according to circulating ESR1 mutation, CA-15.3 and cfDNA increases under first-line anti-aromatase treatment in metastatic breast cancer," Breast Cancer Res., vol. 22, no. 1, May 2020, doi: 10.1186/s13058-020-01290-x.

42. A. Zundelevich et al., "ESR1 mutations are frequent in newly diagnosed metastatic and loco-regional recurrence of endocrine-treated breast cancer and carry worse prognosis," Breast Cancer Res., vol. 22, no. 1, p. 16, Feb. 2020, doi: 10.1186/s13058-020-1246-5.

43. "AR androgen receptor [Homo sapiens (human)] - Gene - NCBI." https://www.ncbi.nlm.nih.gov/gene/367 (accessed Aug. 14, 2020).

44. C. Amaral, T. V. Augusto, M. Almada, S. C. Cunha, G. Correia-da-Silva, and N. Teixeira, "The potential clinical benefit of targeting androgen receptor (AR) in estrogen-receptor positive breast cancer cells treated with Exemestane," Biochim. Biophys. Acta - Mol. Basis Dis., vol. 1866, no. 5, May 2020, doi: 10.1016/j.bbadis.2019.165661.

45. K. H. Kensler et al., "Prognostic and predictive value of androgen receptor expression in postmenopausal women with estrogen receptor-positive breast cancer: Results from the Breast International Group Trial 1-98," Breast Cancer Res., vol. 21, no. 1, p. 30, Feb. 2019, doi: 10.1186/s13058-019-1118-z.

46. H. Qin et al., "The impact of PI3K inhibitors on breast cancer cell and its tumor microenvironment," PeerJ, vol. 2018, no. 6, 2018, doi: 10.7717/peerj.5092.

47. "PIK3CA phosphatidylinositol-4,5-bisphosphate 3-kinase catalytic subunit alpha [Homo sapiens (human)] - Gene - NCBI.” https://www.ncbi.nlm.nih.gov/gene/5290 (accessed Aug. 14, 2020).

48. "PIK3CB phosphatidylinositol-4,5-bisphosphate 3-kinase catalytic subunit beta [Homo sapiens (human)] - Gene - NCBI.” https://www.ncbi.nlm.nih.gov/gene/5291 (accessed Aug. 14, 2020).

49. "PIK3CD phosphatidylinositol-4,5-bisphosphate 3-kinase catalytic subunit delta [Homo sapiens (human)] - Gene - NCBI.” https://www.ncbi.nlm.nih.gov/gene/5293 (accessed Aug. 14, 2020).

50. R. J. Crowder et al., "PIK3CA and PIK3CB inhibition produce synthetic lethality when combined with estrogen deprivation in estrogen receptor-positive breast cancer," Cancer Res., vol. 69, no. 9, pp. 3955-3962, May 2009, doi: 10.1158/0008-5472.CAN-08-4450.

51. D. Huang, L. Tang, F. Yang, J. Jin, and X. Guan, "PIK3CA mutations contribute to fulvestrant resistance in ER-positive breast cancer.," Am. J. Transl. Res., vol. 11, no. 9, pp. 6055-6065, 2019, Accessed: Aug. 12, 2020. [Online]. Available: http://www.ncbi.nlm.nih.gov/pubmed/31632573. 
52. B. Verret, J. Cortes, T. Bachelot, F. Andre, and M. Arnedos, "Efficacy of PI3K inhibitors in advanced breast cancer," Annals of oncology : official journal of the European Society for Medical Oncology, vol. 30, no. 10. NLM (Medline), pp. x12-x20, Dec. 01, 2019, doi: 10.1093/annonc/mdz381.

53. J. S. Chen et al., "PIK3CD induces cell growth and invasion by activating AKT/GSK-3 $\beta / \beta$-catenin signaling in colorectal cancer," Cancer Sci., vol. 110, no. 3, pp. 997-1011, Mar. 2019, doi: $10.1111 /$ cas.13931.

54. E. G. Puigjané, "A new ligand-based approach to virtual screening and profiling of large chemical libraries,” p. 172, 2008.

55. W. Cui, A. Aouidate, S. Wang, Q. Yu, Y. Li, and S. Yuan, "Discovering Anti-Cancer Drugs via Computational Methods," Frontiers in Pharmacology, vol. 11. Frontiers Media S.A., May 20, 2020, doi: 10.3389/fphar.2020.00733.

56. T. Bintener, M. P. Pacheco, and T. Sauter, "Towards the routine use of in silico screenings for drug discovery using metabolic modelling," Biochem. Soc. Trans., vol. 48, no. 3, pp. 955-969, Jun. 2020, doi: 10.1042/BST20190867.

57. Z. Zhang et al., "Overcoming cancer therapeutic bottleneck by drug repurposing," Signal Transduction and Targeted Therapy, vol. 5, no. 1. Springer Nature, Dec. 01, 2020, doi: 10.1038/s41392020-00213-8.

58. A. H. Ananth et al., "Design, Synthesis, and Biological Evaluation of 2-(2-Bromo-3-nitrophenyl)-5phenyl-1,3,4-oxadiazole Derivatives as Possible Anti-Breast Cancer Agents," Chem. Biodivers., vol. 17, no. 2, Feb. 2020, doi: 10.1002/cbdv.201900659.

59. X. Lu et al., "A new class of 1,3,5-triazine-based selective estrogen receptor degraders (SERDs): Lead optimization, molecular docking and dynamic simulation," Bioorg. Chem., vol. 97, Apr. 2020, doi: 10.1016/j.bioorg.2020.103666.

60. N. T. Nguyen et al., "Autodock Vina Adopts More Accurate Binding Poses but Autodock4 Forms Better Binding Affinity," J. Chem. Inf. Model., vol. 60, no. 1, pp. 204-211, Jan. 2020, doi: 10.1021/acs.jcim.9b00778.

61. S. Dallakyan and A. J. Olson, "Small-molecule library screening by docking with PyRx," Methods Mol. Biol., vol. 1263, pp. 243-250, 2015, doi: 10.1007/978-1-4939-2269-7_19.

62. B. H. M. Mooers, "Simplifying and enhancing the use of PyMOL with horizontal scripts," Protein Sci., vol. 25, no. 10, pp. 1873-1882, Oct. 2016, doi: 10.1002/pro.2996.

63. K. Mohanraj et al., "IMPPAT: A curated database of Indian Medicinal Plants, Phytochemistry and Therapeutics," Sci. Rep., vol. 8, no. 1, Dec. 2018, doi: 10.1038/s41598-018-22631-z.

64. P. J et al., "DisGeNET: a comprehensive platform integrating information on human diseaseassociated genes and variants," Nucleic Acids Res., vol. 45, no. D1, 2017, doi: 10.1093/NAR/GKW943.

65. D. Szklarczyk et al., "STRING v11: Protein-protein association networks with increased coverage, supporting functional discovery in genome-wide experimental datasets," Nucleic Acids Res., vol. 47, no. D1, pp. D607-D613, Jan. 2019, doi: 10.1093/nar/gky1131.

66. W. Liu et al., "Discovery, Identification and Comparative Analysis of Non-Specific Lipid Transfer Protein (nsLtp) Family in Solanaceae," Genomics, Proteomics Bioinforma., vol. 8, no. 4, pp. 229-237, Dec. 2010, doi: 10.1016/S1672-0229(10)60024-1.

67. "Vina output analysis using Discovery Studio visualizer - Bioinformatics Review." https://bioinformaticsreview.com/20191101/vina-output-analysis-using-discovery-studio-visualizer/ (accessed Aug. 15, 2020). 